\title{
An unusual cause for fever of unknown origin: Pulmonary arterial aneurysm
}

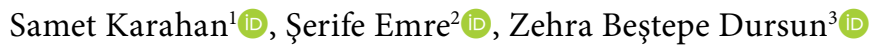 \\ ${ }^{1}$ Department of Internal Medicine, Division of Rheumatology, Kayseri City Training and Research Hospital, Kayseri, Turkey \\ ${ }^{2}$ Department of Internal Medicine, Kayseri City Training and Research Hospital, Kayseri, Turkey \\ ${ }^{3}$ Department of Infectious Diseases and Clinical Microbiology, Kayseri City Training and Research Hospital, Kayseri, Turkey
}

Fever of unknown origin (FUO) in adults is defined as a temperature higher than $\geq 38.3^{\circ} \mathrm{C}$ $\left(101^{\circ} \mathrm{F}\right)$ that lasts for more than three weeks with no obvious source, despite appropriate investigation. ${ }^{1}$ Herein, we present a case with a long febrile period in whom positron emission tomography-computed tomography (PET-CT) had a high maximum standardized uptake $\left(\mathrm{SUV}_{\max }\right)$ nodule and diagnosed as a pulmonary arterial aneurysm (PAA) due to Behçet's syndrome (BS) after a detailed medical history/physical examination.

A 42-year-old female patient was admitted to the hospital with $38.6^{\circ} \mathrm{C}$ fever. Amoxicillin + clavulanate was prescribed due to a possible upper respiratory tract infection. Despite antibiotherapy, febrile period continued. Posteroanterior lung radiograph was performed which revealed no pathology, and the antibiotic was switched to clarithromycin. However, patient's fever persisted. When she applied to the infectious diseases outpatient clinic with $39^{\circ} \mathrm{C}$ of fever, acute phase reactants (APRs) were high (C-reactive protein: $56 \mathrm{mg} / \mathrm{dL}$ ). Urine culture was sterile, and there was no abnormality in lumbar puncture. Due to persistent nonproductive cough, she was prescribed moxifloxacin $400 \mathrm{mg} /$ day once daily. Although the patient used different antibiotics within the past three weeks, her fever continued and APRs were still high. Therefore, she was hospitalized. Aerobic/anaerobic blood cultures were sterile. Epstein-Barr virus, cytomegalovirus, and human immunodeficiency virus serologies were all negative. Abdominopelvic ultrasound was also negative for intra-abdominal abscess, hepatosplenomegaly, and pelvic pathologies. Transesophageal echocardiography revealed no abnormal findings. No tuberculous bacilli were detected by acid-resistant bacilli stain in the sputum and the urine. Gruber-Widal agglutination and Rose Bengal tests were negative. Carbapenem and antifungal drugs were added to the treatment in the second week of hospitalization. Despite these antibiotics and antifungals, her fever persisted up to $39^{\circ} \mathrm{C}$. The patient had oral aphthous lesions for 10 to 12 times per year. During follow-up, thrombophlebitis developed in the vascular access in the left antecubital region. Intravenous

Received: December 27, 2020 Accepted: January 18, 2021 Published online: June 24, 2021

Correspondence: Samet Karahan, MD. Kayseri Şehir Eğitim ve Araştırma Hastanesi, İç Hastalıkları Kliniği, Romatoloji Bölümü, 38080 Kocasinan, Kayseri, Türkiye. Tel: +90 352 - 3157700 e-mail: doktorsamet@yahoo.com

\section{Citation:}

Karahan S, Emre Ş, Beştepe Dursun Z. An unusual cause for fever of unknown origin: Pulmonary arterial aneurysm. Arch Rheumatol $2021 ; 36(4): 620-622$. 


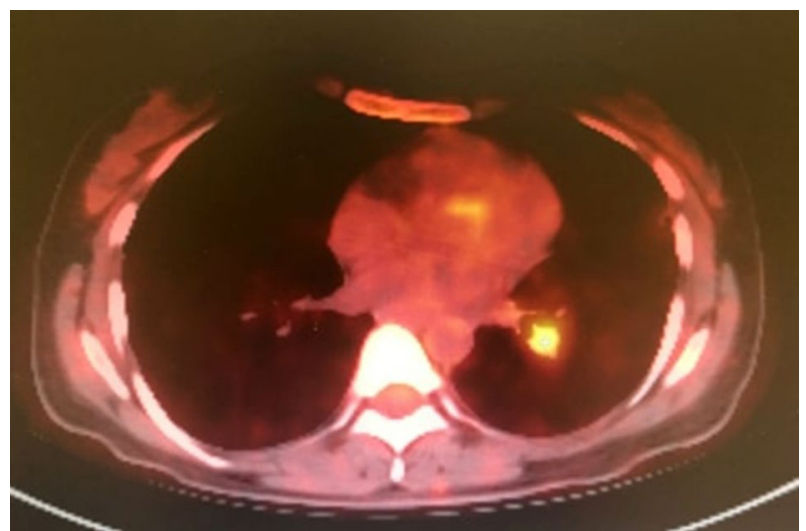

Figure 1. Moderately increased FDG uptake $\left(\mathrm{SUV}_{\max }\right.$ : 1.14) in the lower lobe superior segment of the left lung.

FDG: Fluorodeoxyglucose; $\mathrm{SUV}_{\max }$ : Maximum standardized uptake value.

catheter was revised, thrombophlebitis recurred on the right and confirmed by ultrasonography. After careful examination of perineum, there was a genital scar on the left labium majus. The patient was diagnosed with BS. As the fever is not a common problem in BS, PET-CT was performed to exclude possible malignancies. Moderately increased fluorodeoxyglucose (FDG) uptake (SUV max: 1.14) was observed in the upper lobe posterior and lower lobe superior (Figure 1). Subsequently, methylprednisolone $40 \mathrm{mg} /$ day was initiated. After five days of medium-dose steroid therapy, the patient's APRs started to decrease, thrombophlebitis was cured, and her fever reached normal levels. Based on the PET-CT findings, the patient underwent pulmonary CT angiography and three aneurysmatic dilatations with a diameter of $11 \mathrm{~mm}$ were observed in the left main pulmonary artery (Figure 2). In the light of these findings, the patient was given methylprednisolone $1,000 \mathrm{mg}$ for three days. Cyclophosphamide $1,000 \mathrm{mg} / \mathrm{month}$ was also planned with six cycles. After cyclophosphamide treatment, the patient received low-dose steroid and azathioprine $2 \mathrm{mg} / \mathrm{kg} /$ day and did not experience any fever attacks after approximately nine months of follow-up. No increase in the aneurysm size was detected in CT angiography at one year. A written informed consent was obtained from the patient for all diagnostic and therapeutic procedures.

Fever is rarely reported in BS as an initial presentation. There are case reports describing

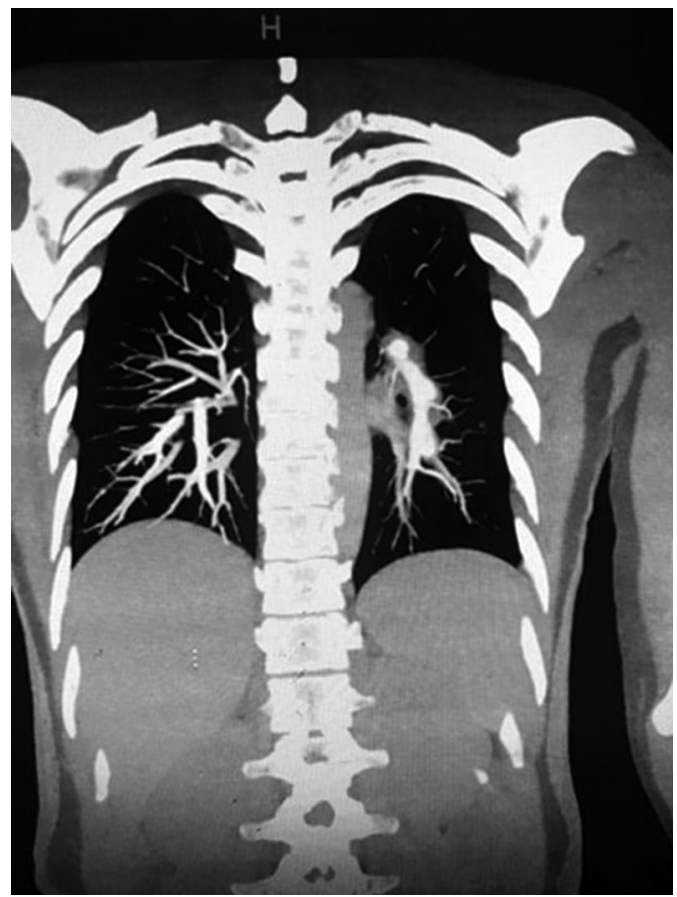

Figure 2. Computed tomography angiography: Soft tissue increase is observed in the left hilar region adjacent to the left pulmonary artery. In addition, three aneurysmatic dilatations with a diameter of $11 \mathrm{~mm}$ are observed in the left pulmonary artery.

fever, particularly in patients with vascular or neurological involvement. Pulmonary arterial aneurysm is one of the typical lesions of BS and the most common form of pulmonary involvement. ${ }^{2}$ It usually occurs in young men, may be single or multiple, unilateral or bilateral, saccular or fusiform, and located in the main pulmonary artery or lobar/segmental arteries. ${ }^{3}$ Pulmonary arterial aneurysms are usually multiple in two or more lobes. According to a report discussing the sexual differences of clinical involvement in BS, vascular involvements were more frequent and severe in men. ${ }^{4}$ Also, according to the data of another report, male sex and an earlier age of onset of the disease were associated with more frequent and more severe vascular involvement in BS. ${ }^{5}$ The fact that our patient was female and relatively old and, moreover, the cause of FUO could not be found after 1.5 months of a detailed investigation, but finally diagnosed with BS based on a meticulous medical history and physical examination makes the case interesting. 
In conclusion, CT has largely replaced conventional angiography in diagnosis, and magnetic resonance imaging is also useful, but less reliable for diagnosing small PAAs. ${ }^{6}$ Among causes of protracted FUO, BS is rare, but should be considered together with the other types of vasculitis. In a febrile patient with suspected or documented BS, a careful investigation for thromboembolism or aneurysm is required after infections are ruled out.

\section{Declaration of conflicting interests}

The authors declared no conflicts of interest with respect to the authorship and/or publication of this article.

\section{Funding}

The authors received no financial support for the research and/or authorship of this article.

\section{REFERENCES}

1. Mulders-Manders C, Simon A, Bleeker-Rovers C. Fever of unknown origin. Clin Med (Lond) 2015;15:280-4.

2. Ozguler Y, Leccese P, Christensen R, Esatoglu SN, Bang D, Bodaghi B, et al. Management of major organ involvement of Behçet's syndrome: A systematic review for update of the EULAR recommendations. Rheumatology (Oxford) 2018;57:2200-12.

3. Aksu K, Koçanaoğullari H, Keser G, Inal V, Kabasakal $\mathrm{Y}$, Oksel $\mathrm{F}$, et al. A case of Behçet's disease with pulmonary arterial aneurysm and secondary amyloidosis. Rheumatology (Oxford) 2002;41:831-2.

4. Ucar-Comlekoglu D, Fox A, Sen HN. Gender differences in Behçet's disease associated uveitis. J Ophthalmol 2014;2014:820710.

5. Balta S, Balta I, Ozturk C, Celik T, Iyisoy A. Behçet's disease and risk of vascular events. Curr Opin Cardiol 2016;31:451-7.

6. Edrees A, Naguib S, El Menyawi M, Ismail I, Nagah H. Pulmonary manifestations in a group of patients with Behcet's disease. Int J Rheum Dis 2017;20:269-75. 